\title{
Políticas públicas para inovação em software: uma abordagem via formação de competências*
}

\author{
Giancarlo Nuti Stefanuto** \\ Angela Maria Alves*** \\ Clenio Figueiredo Salviano ${ }^{* * * *}$ \\ Carolina Vaghetti Mattos ***** \\ Camila Zeitoum ${ }^{* * * * * *}$
}

\section{Resumo}

O artigo apresenta uma abordagem metodológica desenvolvida para avaliar e certificar software resultante de desenvolvimento e inovação tecnológica realizados no país. A análise via formação de competências originou-se a partir de uma demanda do Ministério da Ciência e Tecnologia e Inovação (MCTI) e teve como resultado o desenvolvimento da metodologia Certics. Tal abordagem parte da identificação das competências formadas no entorno do desenvolvimento de um software para identificar seu conteúdo tecnológico e inovativo. Em janeiro de 2014, o Decreto n. 8186 estabeleceu que softwares resultantes de desenvolvimento

* Os autores agradecem o apoio financeiro dos projetos MCTI/01200.001832/2011 - CTENIC e FINEP 0113009300 - Implementação da CERTICS, o trabalho e energia da equipe da CERTICS e dos demais colaboradores do CTI Renato Archer e Facti, e as contribuiçôes e sugestôes dos revisores anônimos da RBI.

** Fundação de Apoio à Capacitação em Tecnologia da Informaçāo (FACTI), Campinas (SP), Brasil. E-mail: giancarlo.stefanuto@ facti.com.br

*** Centro de Tecnologia da Informação Renato Archer (CTI), Campinas (SP), Brasil. E-mail: angela.alves@cti.gov.br

*** Centro de Tecnologia da Informação Renato Archer (CTI), Campinas (SP), Brasil. E-mail: clenio.salviano@cti.gov.br

**** Fundação de Apoio à Capacitação em Tecnologia da Informação (FACTI), Campinas (SP), Brasil. E-mail: carolina.mattos@ facti.com.br

**** Fundação de Apoio à Capacitação em Tecnologia da Informação (FACTI) e Universidade Estadual de Campinas (UNICAMP), Campinas (SP), Brasil. E-mail: camila.zeitoum@facti.com.br 
e inovação tecnológica realizados no país, certificados pela Certics, podem obter margem de preferência de até $18 \%$ em compras governamentais. O artigo conclui que a aplicação prática da Certics mostra-se viável. Foram avaliados e certificados 18 softwares e outros oito estão em fase de certificação, totalizando 22 empresas participantes do setor de software brasileiro.

PALAVRAS-CHAVE | Inovação Tecnológica; Setor Software; Compras Públicas; Política Pública; Avaliação

Códigos JEL | O38

\section{Public policies for software innovation: an approach on competences development}

\section{Abstract}

This paper presents a methodological approach developed for software evaluation and certification resulting from technological development and innovation that occurred in the country. The approach via competency development originated from a demand of the Ministry of Science, Technology and Innovation (MCTI) and resulted in the development of the CERTICS Methodology. This approach consists of the identification of competencies formed during the software development in order to verify its technological and innovative content. In January 2014, Decree No. 8186/14 established the preference margin up to $18 \%$ in public procurements for software certified by CERTICS. The article concludes that the application of CERTICS is feasible with 18 softwares evaluated and certified. Another 8 are in the process of certification. There are 22 companies in the Brazilian software sector that have software certified by CERTICS.

KEYWORDS | Technological Innovation; Software industry; Government Procurement; Public Policy; Assessment

\section{JEL-CODES | O38}




\section{Introdução}

O uso crescente de software nos diversos segmentos produtivos tem levado a um ritmo intenso de desenvolvimento e inovaçōes tecnológicas. O software torna-se cada vez mais complexo e, dada sua intangibilidade, é produzido cada vez mais de forma colaborativa.

Estabelecer uma política pública de incentivo ao desenvolvimento e inovação tecnológica para software não é uma tarefa simples. Crescentemente, os contornos do setor de software tornam-se tênues, diante de sua pervasividade em diversos setores que utilizam soluçôes de software para ampliar sua competitividade.

Um dos desafios para a construção de uma política de incentivo à inovação é a identificação das empresas que acrescentam ao país novos conhecimentos, inovaçōes tecnológicas e novos negócios. Fortalecer essas empresas e, principalmente, os fatores e processos que possibilitam sua existência mostra-se como um alvo relevante a ser perseguido.

Em 2010, o Brasil passou a adotar um instrumento de política pública de margem de preferência em compras públicas, estabelecido pela Lei n. 12.349/10, regulamentada pelo Decreto n. 7.546/11. De acordo com essa Lei, produtos e serviços resultantes de desenvolvimento e inovação tecnológica realizados no país podem obter margens de preferência de até $25 \%$ em compras governamentais. A margem de preferência foi estabelecida para um conjunto de setores, entre estes o de software. E coube aos Ministérios de Ciência, Tecnologia e Inovação (MCTI) e do Desenvolvimento, Indústria e Comércio Exterior (MDIC) a definição de requisitos e critérios para a aplicação destas margens.

Em 2011, o MCTI demandou ao Centro de Tecnologia da Informação Renato Archer (CTI Renato Archer), o desenvolvimento de uma metodologia para avaliar software resultante de desenvolvimento e inovação tecnológica realizados no país.

Em junho de 2013, foi lançada a Portaria n. 555 do MCTI, definindo a metodologia Certics como o conjunto de requisitos e critérios para certificar software resultante de desenvolvimento e inovação tecnológica realizados no Brasil. Em janeiro de 2014, foi emitido o Decreto n. 8.186, estabelecendo a margem de preferência de até $18 \%$ para compras públicas de softwares com a certificação Certics.

A aplicação de margem de preferência pelo governo brasileiro assemelha-se a políticas públicas adotadas em outros países, que também utilizam as compras públicas para estimular as empresas nacionais a investirem em tecnologia e inovação e para formar capacidades tecnológicas e produtivas locais. Apesar de apresentarem 
diferentes formas de aplicação, as políticas de compras públicas nos diversos países têm em comum a necessidade de identificar e diferenciar um produto produzido localmente, seja em decorrência de suas próprias características, seja pelo perfil da empresa que o produz.

Alguns tipos de abordagens vêm sendo desenvolvidos e aplicados para dar suporte ao mecanismo de preferência em compras públicas para produtos e/ou empresas. Há abordagens baseadas na análise do capital majoritário da empresa fornecedora do produto, resultando na seleção de empresas cujo capital majoritário é nacional. Outras baseiam-se na exigência da existência de planta produtiva da empresa no território onde está sendo aplicada a margem de preferência. Há ainda aquelas que privilegiam aspectos relacionados diretamente ao produto, tais como a qualidade, que é verificada a partir de avaliaçôes que resultam em certificações de qualidade.

Porém, a utilização destes tipos de abordagens para a diferenciação e preferência de produtos com significativo grau de intangibilidade como o software é praticamente inviável. Os produtos intangíveis, ${ }^{1}$ em especial o software, pela sua natureza intrínseca, podem ser desenvolvidos por colaboradores distribuídos globalmente, desde que suportados por processos e plataformas que organizem esta produção.

Diante deste cenário e da demanda colocada pelo governo brasileiro, o desafio foi pensar qual seria o tipo de abordagem adequada e viável para avaliar e dar preferência a um software que traz desenvolvimento tecnológico e inovação para o país, levando-se em consideração os custos, tempo necessário e suficiência de critérios para aplicação desta abordagem.

O presente trabalho tem como objetivo apresentar a abordagem via formação de competências e sua utilização para o desenvolvimento da metodologia Certics. Também será apresentada a aplicação prática desta metodologia como instrumento de política pública de margem de preferência em compras públicas no setor de software brasileiro, mostrando suas evidências empíricas. A abordagem via formação de competências foi desenvolvida com foco nas competências formadas no entorno do desenvolvimento de um software, e que resultam na geração local de tecnologia e inovaçōes tecnológicas.

O artigo está dividido em cinco seções, incluindo esta introdução. A seguir apresenta-se um breve panorama do uso de compras públicas para o desenvolvimento

\footnotetext{
1 A utilização da denominação produtos intangíveis por vezes é inadequada, pois a natureza de um bem intangível assemelha-se mais a um serviço. No caso do software, há situaçôes em que a natureza de serviços predomina, como, por exemplo, plataformas de software em nuvem que fornecem serviços: Google, Wikipedia, entre outros. Porém, como usualmente software é abordado como produto, neste trabalho será utilizada a denominação de produtos intangíveis, para facilitar o desenvolvimento do texto.
} 
da indústria de software no Brasil e algumas referências da experiência internacional. Posteriormente, é mostrada a abordagem via formação de competências como eixo para avaliação do conteúdo tecnológico e inovativo de um software. O trabalho também apresenta a metodologia Certics, sua aplicação prática, benefícios, limitações e, por fim, as considerações finais.

\section{Uso do poder de compras no Brasil e a experiência internacional}

No Brasil, o uso do poder de compras públicas para estimular o desenvolvimento do setor de software no Brasil é um fato recente na sua história. Até a promulgação da Lei n. 12.349/10, não havia garantia de compra governamental para produtos inovadores e com densidade tecnológica para o setor de software. O Brasil, apesar de se configurar como um dos maiores mercados mundiais de software, ainda apresenta-se predominantemente como consumidor desse produto. A própria regulamentação da Lei n. 12.349/10, que abrange diferentes setores por meio de decretos e instruçôes normativas, e as dificuldades para adequar estes instrumentos às especificidades do setor de software no Brasil indicam a existência de uma cultura de uso de compras públicas para software bastante incipiente. ${ }^{2}$

A opção do governo brasileiro para a introdução de margem de preferência para um conjunto de setores considerados estratégicos, entre estes o de software, focalizou-se na identificação de produtos resultantes de desenvolvimento e inovação tecnológica realizados no país. Trata-se, portanto, de uma abordagem focalizada nas características intrínsecas do produto. Em consonância, analisando a exposição de motivos da Lei n. 12.349/2010 e do Decreto n. 7.546/11, observou-se que os objetivos maiores relacionados com a implantação da margem de preferência vinculavam-se ao aumento da autonomia tecnológica, da capacidade inovativa e da consecução de negócios para fomentar o desenvolvimento sustentável do país.

No cenário internacional, foram encontradas algumas experiências que se assemelham ao caso brasileiro no que se refere ao uso do poder de compras públicas para estímulo ao desenvolvimento setorial, mas com diferentes abordagens.

Vários casos observados dão preferência a produtos e/ou empresas nacionais, entendendo-se implicitamente, como empresas nacionais, aquelas cujo capital majoritário é nacional e, como produtos nacionais, aqueles que são produzidos pelas empresas nacionais.

2 Parte dos autores participou de discussōes e análises de dados no Ministério da Fazenda e no Ministério da Ciência, Tecnologia e Inovação para a conformação dos referidos instrumentos de regulamentação. 
Países como Turquia, Canadá, Austrália e Nova Zelândia utilizam margem de preferência para produtos nacionais (SSENNOGA, 2006). No caso da Turquia, a legislação local estabeleceu um conselho independente para supervisionar licitações públicas das quais empresas estrangeiras localizadas no país podem participar. A lei oferece uma margem de preferência de $15 \%$ para licitantes nacionais, indisponível caso estabeleçam joint venture com licitantes estrangeiros. A Turquia estendeu a definição de licitante doméstico a corporações de propriedade estrangeira estabelecidas sob a lei turca (USTR, 2010). Omã, por sua vez, oferece margem de preferência de $10 \%$ às empresas omanis nas compras governamentais (USTR, 2000).

A República Tcheca também utiliza margem de preferência em casos de propostas de fornecedores nacionais sobre fornecedores estrangeiros localizados no país, desde que o custo adicional não ultrapasse 10\% (JOUMARD, 2004).

Outras abordagens baseiam-se na exigência de que o bem seja produzido em uma planta produtiva localizada no país onde está sendo aplicada a margem de preferência. É o caso de bens domésticos nos Estados Unidos. Já em 1933 foi aprovado pelo Congresso norte-americano o Buy American Act, que oferece uma margem de preferência de $6 \%$ a fornecedores de bens domésticos, podendo alcançar $12 \%$ no caso de pequenas empresas em regiōes de altos índices de desemprego e 50\% para equipamento militar.

Nos países citados anteriormente, as compras públicas incentivadas pelo governo não foram adotadas apenas para o setor de software, sendo de caráter geral. Os únicos casos encontrados no cenário internacional que explicitam regras de compras públicas específicas para o setor de software foram a China e a Coreia do Sul.

Observa-se, no caso chinês, uma abordagem própria que define o conceito de produto doméstico. A lei de compras governamentais na China entrou em vigor em 2002 e define que as agências governamentais devem adquirir apenas produtos e serviços de software domésticos. Somente em caso de dispensa especial do Ministério da Indústria da Informação e do Ministério das Finanças é que as empresas chinesas podem comprar produtos de software não domésticos. Para ser considerado um produto doméstico, o software deve atender aos seguintes requisitos: ser desenvolvido dentro da China; a licença ser de propriedade de uma pessoa física, pessoa jurídica ou outras organizaçóes de dentro da China; e o custo de desenvolvimento incorrido na China deve contabilizar pelo menos $50 \%$ do custo total do software (SAXENIAN, 2003).

$\mathrm{Na}$ Coreia do Sul existe um marco legal com o objetivo específico de impulsionar a indústria de software como parte do desenvolvimento industrial do 
país, o Software Industry Promotion Act, de 2000. Entre as principais linhas dessa lei, estão criação e desenvolvimento da infraestrutura necessária para viabilizar o crescimento do setor, promoção do desenvolvimento de tecnologias, padronização e certificações de qualidade de produto e processo. Há dois certificados de qualidade especialmente direcionados às empresas atuantes no mercado doméstico, os quais objetivam alavancar a qualidade dos softwares produzidos na Coreia do Sul, principalmente para as pequenas e médias empresas, que somam 99\% dos casos (SEOK-KYOO, 2011). O certificado Good Software, emitido pelos laboratórios da Telecom Technology Association (TTA), é apenas para produto (software) da empresa, ${ }^{3}$ e começou a ser utilizado como padrão a partir do momento que as autoridades de compras públicas do país adotaram-no como critério de escolha em relação a outros softwares (SUNG-MI, 2012). O certificado Software Process, também emitido pelo TTA, mede a qualidade no processo de produção de softwares seguindo parâmetros baseados na norma ISO/IEC 15504 e nos modelos do CMMI. ${ }^{4} \mathrm{O}$ Ministério de Defesa sul coreano utiliza este certificado como referência em suas licitações de softwares, e pretende apoiar as empresas potenciais na obtenção do certificado (CHUNG, 2013). Portanto, verifica-se aqui uma abordagem voltada à seleção pela qualidade do software produzido.

A partir do observado no cenário internacional, verificou-se que o tipo de demanda proposta pela Lei n. 12.349/10 e pelo Decreto n. 7.546/11 apresentava características próprias, endereçando uma abordagem voltada para a análise do conteúdo tecnológico e inovativo do software, ao contrário do praticado na maior parte dos países. Concluiu-se que a solução a ser desenvolvida deveria buscar uma abordagem inovadora. O desafio então configurou-se em criar uma abordagem específica para saber se um software teve seu desenvolvimento e inovação tecnológica realizados no País.

\section{Abordagem via formação de competências}

A ausência de referências internacionais exatamente iguais ao caso brasileiro levou à busca de uma solução que considerasse a natureza particular do software. De

3 O certificado é baseado em seis características de qualidade: funcionalidade, confiabilidade, usabilidade, eficiência e capacidade de manutenção.

4 O certificado é baseado em três níveis de maturidade, em vez dos cinco do CMMI: a passagem do nível 1 (inicial, onde os processos não se encontram estruturados) para o nível 2 contempla melhoria do processo no nível do projeto. A passagem do nível 2 para o nível 3 contempla a melhoria do processo no nível da organização da empresa (onde há métricas para medir todos os projetos da empresa, conjuntamente) (HWANG; IM, 2011). 
fato, a natureza intangível e pervasiva do software lhe confere características muito particulares, quando comparado a outros produtos. Além disso, o software é utilizado por diversos setores para aprimorar seus processos produtivos, redesenhar e inovar produtos, chegar a novas soluções tecnológicas, o que o torna de extrema importância estratégica. Como exemplo, temos o setor financeiro, no qual diversos tipos de cálculos e transações somente se tornam possíveis a partir dos avanços da tecnologia de software.

Por isso, a avaliação do objeto software é muito particular. Há alguns anos tornou-se relativamente fácil produzir software a partir de colaboradores espalhados em diversas partes do mundo, bem como construir software a partir de componentes preexistentes.

Além de redefinir outros produtos, a tecnologia de software redefine a si própria continuamente. Portanto, uma abordagem usual para identificar produtos com tecnologia e inovaçôes tecnológicas locais a partir da análise de seus componentes (por exemplo, um carro ou um equipamento médico) seria de difícil aplicação para software.

Uma estratégia de avaliar e dar preferência a um software por meio da análise da origem das linhas de código-fonte e módulos que o compõem ou das etapas de sua produção seria uma tarefa que demandaria longo tempo de análise e seria, portanto, praticamente impossível para softwares complexos, com milhôes de linhas de códigos e altamente modularizados.

Nesse contexto, a construção da abordagem via formação de competências utilizou uma metodologia de pesquisa denominada pesquisa-ação, em que a construção de uma hipótese de trabalho não se dá a partir de um marco conceitual estabelecido, mas sim do levantamento de literatura e de bases de conhecimento que são cotejados com a experiência prática ou experimental acerca de determinado tema.

Uma das bases de conhecimentos empregada foi a norma ISO/IEC 15504: Tecnologia de Informação - Avaliação de Processos (Process Assessment) (ISO/IEC, 2003; 2008). A norma define uma estrutura de medição da capacidade de processo e requisitos para modelos de referência e métodos de avaliação de processo. A partir desta norma e da experiência do CTI Renato Archer na sua utilização, o caminho encontrado para analisar o conteúdo tecnológico e inovativo de um software foi a avaliação dos processos no entorno do desenvolvimento do software e não o software em si ou sua cadeia produtiva. A construção da abordagem com o uso da Norma ISO/IEC 15504 demandou a construção de uma arquitetura em camadas que orienta a avaliação dos processos (SALVIANO et al., 2008). 
O desafio, então, se focalizou em identificar quais aspectos poderiam ser avaliados objetivamente nos processos que envolvem o desenvolvimento de um software. Partiu-se, assim, da busca e entendimento de capacidades tecnológicas, o que derivou, posteriormente, para competências.

A seguir, apresentam-se o desenvolvimento conceitual realizado para compor a nova abordagem e, posteriormente, a metodologia Certics: competência e áreas de competência.

\subsection{0 desenvolvimento do conceito de competência}

Os resultados tecnológicos e de inovação de uma empresa costumam acontecer na razão direta das capacidades nela existentes. Porém, muitos estudos têm avaliado quais tipos de capacidades são necessários e qual a conjugação necessária destas capacidades para se chegar a esses resultados, pois é frequente que empresas com capacidades técnicas reconhecidas não sejam necessariamente bem-sucedidas no mercado (TEECE, 1986; RUSH et al., 2007).

Alguns estudos são apresentados a seguir, pois foram utilizados para construir a base dos tipos de capacidades necessários para a geração de tecnologia e inovações.

Para Panda e Ramanahtam (1997), as capacidades tecnológicas de uma empresa são o conjunto de habilidades que resultam na adição de valor e inovações, sendo classificadas em:

- capacidades estratégicas - aquelas relacionadas à produção tecnológica e ampliação da base de conhecimentos da empresa, tais como capacidade de criação, design, engenharia, etc.;

- capacidades táticas - aquelas relacionadas à operacionalização da tecnologia e sua comercialização, tais como capacidades de marketing, de vendas, de suporte ao usuário, etc.;

- capacidades suplementares - aquelas que suportam a realização dos dois primeiros grupos, tais como planejamento, melhoria de processo, treinamento, gestão da informação, etc.

A evolução da análise de capacidades técnicas como boas práticas para a geração de tecnologia e inovação levou à construção de modelos de avaliação de processo para atividades de pesquisa e desenvolvimento (P\&D) e inovação, ou seja, modelos para a análise do grau de maturidade de uma organização em relação a essas práticas. 
Um dos modelos (BERG et al., 2002; 2004) indica boas práticas de P\&D agrupadas em nove categorias, incluindo: estratégias tecnológicas bem planificadas e claras; gestão do portfólio tecnológico; monitoramento das tecnologias estratégicas para o produto; planejamento das atividades de P\&D com metas e objetivos, entre outras.

Destas referências depreende-se a existência de duas naturezas de capacidades: as diretamente relacionadas à produção da tecnologia; e as que dão suporte e potencializam as primeiras. Também depreende-se que o desenvolvimento e a inovação tecnológica, para que não sejam atividades fortuitas e pontuais, devem ser tratados como processos.

De fato, a capacidade de inovar é um extenso processo de aprendizagem, que gradualmente acumula procedimentos, rotinas, até que se tornem práticas internas à organização (RUSH et al, 2007). Estas práticas conformam uma cultura de geração de tecnologia e de inovação dentro da organização, que, ao mesmo tempo, são fruto de capacidades existentes e que as retroalimentam, também estimulando a criação de novas capacidades.

Embora os termos geração de tecnologia, atividades de P\&D e inovações sejam abordados conjuntamente ou alternadamente, entende-se que as capacidades a eles relacionadas não são necessariamente as mesmas; algumas vezes são distintas e complementares e outras vezes muito específicas a cada uma destas dimensões.

Também considera-se que a simples existência de capacidades tecnológicas - seja parcialmente (capacidades centradas do desenvolvimento de tecnologia), seja um conjunto de capacidades complementares (gestão de tecnologia, negócios, etc.) - não implica necessariamente um impacto positivo no desempenho da empresa em termos da geração e comercialização de inovaçôes tecnológicas, pois há possibilidades da ocorrência de externalidades desfavoráveis (política cambial, retração de mercado, etc.).

A busca do entendimento das formas de utilização de capacidades conduziu aos trabalhos de David Teece (1986, 2007), Maria Teresa Fleury e Afonso Fleury (2001, 2004) e Prahalad e Hamel (1990), que forneceram elementos para o uso dinâmico das capacidades tecnológicas.

Para Teece (2007), capacidades dinâmicas são aquelas que podem ser acessadas continuamente para criar, aumentar, aprimorar e proteger a base de bens de uma empresa, podendo ser desagregadas em três tipos de capacidades: de identificar e equacionar oportunidades e desafios; de apoderar-se de oportunidades; e de manter a competitividade por meio da expansão, combinação, proteção e mesmo reconfi- 
guração dos bens tangíveis e intangíveis de seu negócio. As capacidades dinâmicas orquestram as demais capacidades, de modo que a empresa possa "sentir" o mercado e identificar oportunidades, apoderar-se delas e gerenciar desafios e mudanças (TEECE, 2007).

Porém, como identificar e avaliar a maneira como se dá esta orquestração de capacidades no desenvolvimento de um produto?

Para Prahalad e Hamel (1990), a capacidade de combinar, misturar e integrar recursos em produtos e serviços denomina-se competência e, para ser essencial (core competencies), não precisa necessariamente ser baseada em tecnologia stricto sensu: ela pode estar associada ao domínio de qualquer estágio do ciclo de negócios, como por exemplo um profundo conhecimento das condições de operação de mercados específicos.

A partir das considerações anteriores e da definição de competência adotada por Fleury e Fleury (2001) para indivíduos, derivou-se que a orquestração de capacidades para determinado objetivo é definida como uma competência que a empresa possui. O conceito definido foi: competência é a capacidade de mobilizar, integrar e transferir conhecimentos, recursos e habilidades. Esta definição não diz respeito a competências individuais, mas sim organizacionais.

Portanto, ter competência significa não apenas ter uma base de conhecimentos especializados, habilidades e recursos, mas também manter e ampliar esta base, mobilizá-la para determinados fins e, quando necessário, disseminar, transferir esta base. A identificação de competências em uma unidade organizacional se dá pela constatação da existência de um círculo virtuoso e dinâmico de uso de suas capacidades (recursos humanos, processos e conhecimentos) para a geração de tecnologia, inovações e negócios. Sabe-se que esses conhecimentos e habilidades estão presentes nas empresas em formatos diversificados: em pessoas, em documentos ou na definição de processos. Assim, uma vez definido um conceito para competência, a questão prática passou a ser identificar e mensurar esta competência: como identificar as competências que contribuíram para o desenvolvimento de um software de modo a torná-lo inovador? Quais competências foram essenciais para isso?

\subsection{Avaliação e medição das competências}

Além dos trabalhos já citados, também foram utilizados como referência para definir os tipos de competências a serem avaliadas os estudos de Cunha (2005), Rush et al. (2007) e o modelo Enterprise Spice (2010). Nessas análises, evidenciou-se a 
necessidade de se obterem competências complementares às tecnológicas para que uma unidade organizacional inove tecnologicamente. Apesar de terem natureza distinta, por exemplo, tecnologia e negócios, nas pesquisas de campo, verificou-se que há uma intersecção e um intercâmbio entre essas competências. É comum ex-desenvolvedores de software, ou mesmo desenvolvedores ativos, trabalharem na área de vendas. Portanto, são competências aparentemente distintas, mas que se correlacionam todo o tempo para a consecução de objetivos

A partir das reflexões apresentadas no item anterior, apontando a insuficiência das capacidades tecnológicas para consecução das inovações tecnológicas, e da análise da exposição de motivos da Lei n. 12.349/10 e do Decreto n. 7.546/11, que definiram os objetivos macro de aplicação das margens de preferência (autonomia, capacidade inovativa e negócios), foram definidos dois grupos de competências para avaliação de um software:

- competências tecnológicas - conjunto de conhecimentos e habilidades de uma unidade organizacional utilizados para criar ou atualizar uma tecnologia em seus princípios ou funcionalidades;

- competências correlatas - conjunto de conhecimentos e habilidades complementares às competências tecnológicas que, simultaneamente, as potencializam ou são por elas potencializados e que são necessários para a consecução de negócios baseados em conhecimento e para o aumento da capacidade inovativa.

Para precisar os tipos de competências tecnológicas e correlatas que direcionariam a avaliação, houve um novo processo de consulta à literatura e a formulação de uma proposta de tipos de competências e interação com stakeholders para aprimorar o conjunto definido.

Para construir os tipos de competências, partiu-se do trabalho de Cunha (2005), que apresenta cinco categorias de boas práticas para a inovação:

- estratégia e posicionamento de mercado - engloba objetivos, finalidades, metas, diretrizes, políticas, posicionamento de mercado e planos que visam guiar o comportamento global da empresa;

- estrutura e ambiente organizacional interno - refere-se à estrutura organizacional e à organização interna da empresa em relação a suas atividades, processos e projetos;

- gestão de tecnologia - engloba todas as práticas relacionadas à pesquisa e ao desenvolvimento, bem como a aquisição e utilização de novas tecnologias, produtos ou serviços; 
- gestão de pessoas - compreende todas as práticas relacionadas às pessoas, ao recrutamento e seleção, à organização do trabalho, à formação do colaborador e sua remuneração;

- gestão de parcerias - refere-se às parcerias estabelecidas com fornecedores, outras empresas e clientes, instituiçóes de pesquisa, entre outros.

Rush et al. (2007) apontam nove componentes para a medição de capacidades tecnológicas em empresas, entre os quais se destacam: o desenvolvimento de uma estratégia tecnológica; a avaliação e exploração do leque de tecnologias disponíveis; a implementação, absorção e operação da tecnologia dentro da empresa; os processos de aprendizado para a formação de competências tecnológicas; e as parcerias externas. Foram também utilizadas referências do modelo Entreprise Spice (2010), que define 29 processos organizados em quatro categorias. Cada processo define um propósito e um conjunto de melhores práticas.

A partir destas referências foi elaborada uma proposta preliminar de um modelo de referência para avaliação com 12 tipos de competências, que posteriormente foram definidas como áreas de competência, testados em entrevistas de campo com empresas de software e em painéis de especialistas.

O resultado desta pesquisa de campo orientou a redefinição das áreas de competências em quatro áreas:

- desenvolvimento tecnológico - caracteriza-se por competências relacionadas ao desenvolvimento das tecnologias relevantes do software, principalmente nos seus requisitos e arquitetura;

- gestão de tecnologia - cobre a utilização de resultados de pesquisa e desenvolvimento, a apropriação das tecnologias relevantes, a introdução de inovaçôes tecnológicas e a capacidade decisória sobre as tecnologias relevantes;

- gestão de negócios - cobre ações para monitoramento do mercado, ações para antecipação e atendimento de necessidades dos clientes e a evolução do negócio relacionado ao software;

- melhoria contínua - cobre a gestão de pessoas, processos e conhecimento para apoiar e potencializar o desenvolvimento tecnológico, a gestão da tecnologia e a gestão de negócios.

Embora tratadas como áreas individualizadas, com resultados e evidências específicos, observam-se forte imbricação entre estas áreas, influência mútua e trabalho conjunto entre as competências. 
Giancarlo Nuti Stefanuto, Angela Maria Alves, Clenio Figueiredo Salviano, Carolina Vaghetti Mattos, Camila Zeitoum

A Figura 1 resume os principais elementos da abordagem via formação de competências.

\section{FIGURA 1}

Avaliação por áreas de competência da metodologia Certics

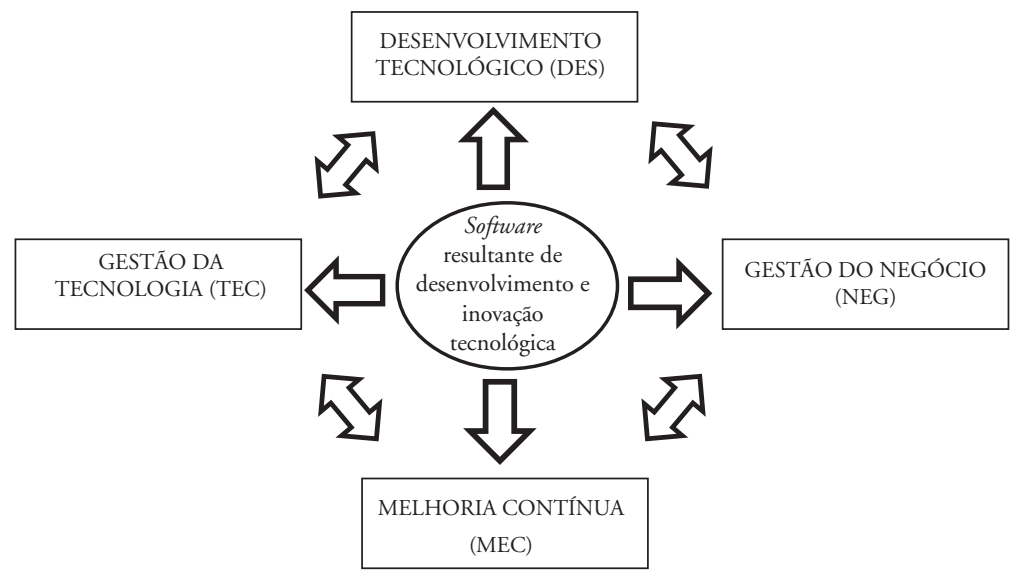

Fonte: Elaboração dos autores.

\section{Aplicação da abordagem para a construção da metodologia Certics}

O desenvolvimento da metodologia Certics foi baseado na abordagem via formação de competências. As áreas de competências definidas - desenvolvimento tecnológico, gestão do negócio, melhoria contínua e gestão tecnológica - forneceram os principais elementos para a construção da metodologia (CTI RENATO ARCHER, 2013; ALVES et al., 2014). Tal construção se deu a partir do uso destes elementos em uma estrutura lógica, de maneira que pudesse traduzir uma metodologia de avaliação de processos no entorno do software.

A estrutura lógica foi definida em quatro camadas hierárquicas, formuladas a partir dos requisitos da Norma ISO/IEC 15504. A Figura 2 apresenta a estrutura lógica da Certics, já incorporando os elementos da abordagem via formação de competências.

Os elementos que compõem a estrutura lógica da Certics são:

- $1^{a}$ camada - conceito fundamental: define o problema a ser resolvido que orienta a avaliação, neste caso, a necessidade de se identificar software resultante de desenvolvimento e inovação tecnológica realizados no país;

- $\quad 2^{\mathrm{a}}$ camada - áreas de competência: definem quais os temas e perguntas-chave direcionam a resolução do problema, neste caso, as quatro áreas de 
competências definidas. A demonstração das competências nestas quatro áreas indicam que o software é resultante de desenvolvimento e inovação tecnológica realizados no Brasil;

- $\quad 3^{a}$ camada - resultados esperados: definem quais os resultados esperados são verificados para confirmar a existência das competências;

- $\quad 4^{a}$ camada - orientações e indicadores: definem as orientações e indicadores que atestam as evidências e comprovam os resultados.

FIGURA 2

Estrutura lógica da metodologia Certics

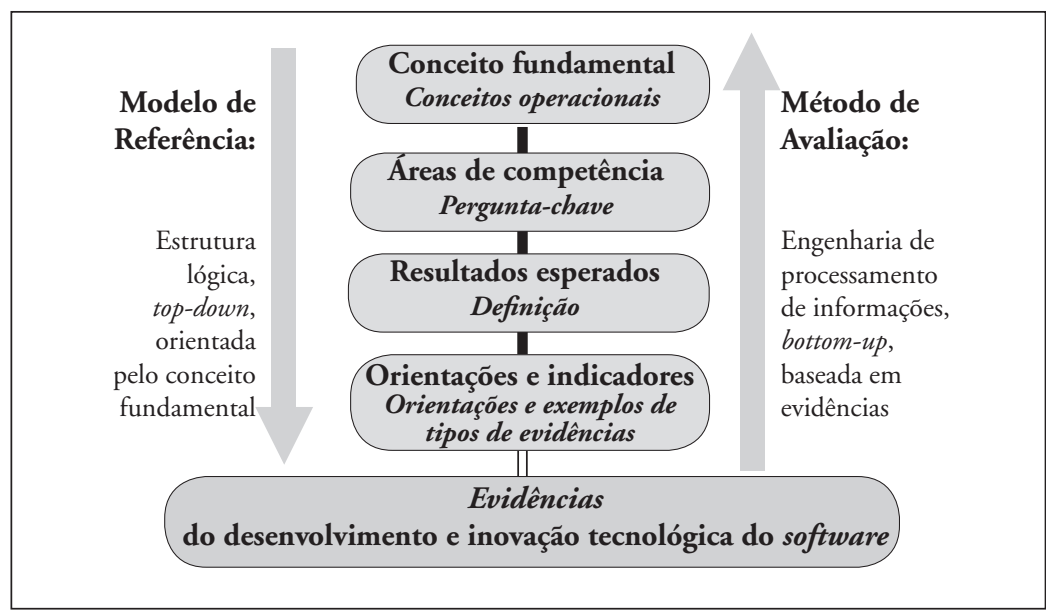

Fonte: Elaboração dos autores.

A estrutura lógica da Certics tem dupla funcionalidade:

- em uma abordagem top-down, ela orienta o avaliador quanto ao modelo lógico para a avaliação, estabelecendo o que está sendo buscado, as perguntas-chave que orientam a avaliação, os resultados que precisam ser verificados, etc.;

- em uma abordagem bottom-up, ela norteia a utilização desta estrutura em uma avaliação. A partir da verificação das evidências, comprovam-se resultados esperados, as competências e o conceito fundamental.

A aplicação dos elementos da abordagem via formação de competências na $1^{\mathrm{a}}$ e $2^{\mathrm{a}}$ camadas orientou a construção dos resultados esperados e das orientações e indicadores, a qual se deu por sucessivas interaçôes com os principais stakeholders.

A partir das referências utilizadas para a construção da abordagem, foi elaborada uma lista de resultados esperados, baseados em boas práticas e capacidades 
técnicas necessárias para a consecução do desenvolvimento tecnológico e/ou inovação tecnológica. ${ }^{5}$ Para cada área de competência definiu-se um conjunto de resultados, que foram testados em aproximadamente 20 empresas de software. A partir daí foi definida a metodologia, apresentada em um painel com 60 especialistas. Com os resultados deste painel elaborou-se a versão 1.0 da metodologia, que foi colocada em consulta pública de agosto a dezembro de 2012. Nesta consulta foram recebidas 333 contribuições para melhoria da metodologia. Além disso, foi realizada uma pesquisa estruturada com 49 micro e pequenas empresas (MPE) de software. A partir dos resultados da consulta pública e da pesquisa com MPE, chegou-se à versão 1.1 da metodologia Certics. Os resultados esperados por áreas de competência são apresentados na Figura 3. Mais detalhes podem ser encontrados no documento da metodologia Certics, no site oficial da certificação. ${ }^{6}$

Figura 3

Resultados esperados na metodologia Certics

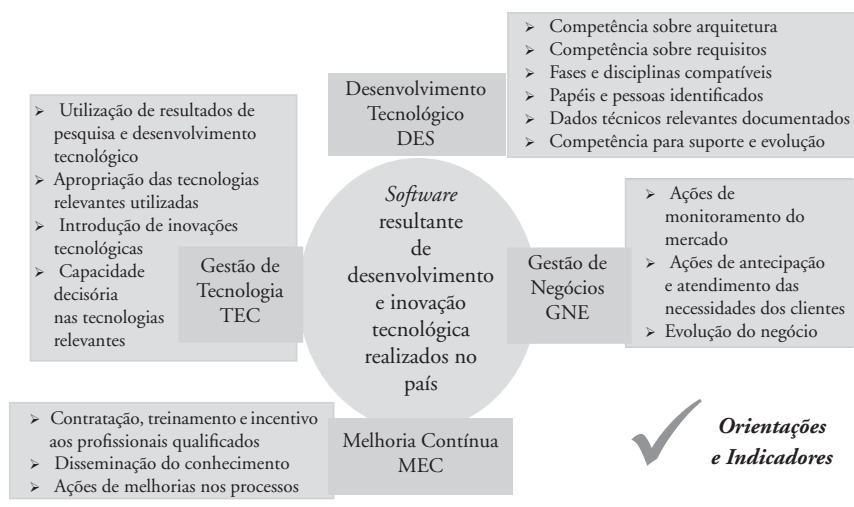

Fonte: Elaboração dos autores.

\subsection{Metodologia Certics: aplicação, benefícios e limitações}

A avaliação de um software na certificação Certics se dá a partir da identificação de evidências que comprovem os resultados esperados. Se os resultados estão presentes, então, comprova-se a existência de competências geradas e, por consequência, constata-se que o software é resultante de desenvolvimento e inovação tecnológica realizados no Brasil.

5 Alguns exemplos que fizeram parte da lista inicial de resultados são a utilizaçăo de ferramentas para o planejamento da tecnologia, como roadmaps, monitoramento do estado da arte da tecnologia, análise de produtos concorrentes, a capacidade de articular e estabelecer parcerias com fornecedores ou com instituiçōes de pesquisa, etc.

6 Disponível em <www.certics.cti.gov.br>. 
Um dos 16 resultados esperados, conforme a Figura 3, a ser comprovado é a competência sobre a arquitetura do software (desenvolvimento tecnológico DES). Para isso, são indicadas na metodologia as evidências a serem verificadas, por exemplo:

- existência de um documento de arquitetura do software, verificando se as pessoas que fizeram este documento ainda permanecem na empresa;

- demonstrar que tem capacidade para fazer alterações nesta arquitetura (verificação de mudanças recentes, etc.).

Outro exemplo de resultado esperado a ser comprovado é a utilização de resultados de pesquisa e desenvolvimento tecnológico (gestão da tecnologia-TEC) no software, seja no seu desenvolvimento ou no seu aprimoramento. Não é exigido que haja na empresa uma unidade de $P \& D$ estruturada para o aprimoramento de determinado software, mas sim a existência de uma cultura de buscar e absorver resultados de P\&D para a solução de desafios tecnológicos apresentados na evolução do software. Neste caso são buscadas evidências que comprovem o desafio tecnológico identificado e a solução encontrada por meio do uso de atividades de P\&D ou por meio de parceria com institutos de pesquisa ou universidades. Esta solução tem que ter sido implementada no software.

Portanto, resumidamente, a aplicação da metodologia Certics consiste em verificar 16 resultados esperados, a partir de uma lista indicativa de exemplos de evidências, seguindo uma estrutura lógica definida.

Para cada resultado esperado há um conjunto específico de orientações e uma lista de exemplos de evidências. Entretanto, no processo de construção da metodologia, verificamos que as quatro áreas de competências usualmente se sobrepõem, especialmente em micro e pequenas empresas, onde é comum, por exemplo, que o desenvolvedor de software também seja a interface de vendas da empresa. Portanto, a metodologia também prevê que um mesmo conjunto de evidências possa comprovar mais do que um resultado esperado.

A avaliação de processos no entorno do software que embasa a metodologia Certics permite grande flexibilidade na sua aplicação. Ao se deslocar o foco da avaliação para as competências formadas em seu entorno, em vez da avaliação dos componentes e das funcionalidades do software, diminui-se drasticamente o tempo de avaliação, pois já não é necessária uma avaliação técnica especializada e não há uma dependência do tempo de avaliação com relação ao tamanho do software. 
A existência de uma lista indicativa e não exaustiva de evidências permite que empresas de diferentes portes possam disponibilizar evidências de formatos e conteúdos muito distintos e, que apesar disso, atendem à metodologia. Por exemplo, o resultado esperado evolução do negócio (gestão de negócios - GNE) associado ao software em uma grande empresa pode ser evidenciado por estudos de mercado, estudos especializados, etc., enquanto em uma pequena empresa pode ser observado por contratos ou parcerias estabelecidas com clientes e fornecedores.

O foco da avaliação em processos no entorno do software também permite que seja identificada a cultura de uso de P\&D e de geração de inovaçôes, ${ }^{7}$ mesmo que sejam pouco estruturadas, pois são avaliados os processos relativos a estas dimensões e não somente os resultados obtidos.

Porém, a definição das áreas de competência tem uma dependência temporal, ou seja, a partir da sua aplicação das preferências em compras, espera-se um aumento do grau de competência nas áreas definidas e que novas áreas de competência sejam incluídas em função do aumento da maturidade do setor. Por exemplo, em uma indústria de software com maior grau de maturidade, com inserção internacional, são esperadas outras áreas de competência, como gestão de parceria e alianças, gestão de riscos, gestão de projetos, gestão de P\&D\&I, etc.

A metodologia Certics não se aplica a produtos de software ainda não desenvolvidos e, por isso, não é aplicável a boa parte das empresas em incubadoras ou startups, pois usualmente este tipo de empresas possui softwares ainda em desenvolvimento e não comercializados. E, apesar de detectar a cultura de $\mathrm{P} \& \mathrm{D}$ relacionadas ao software, o grau de maturidade ainda bastante incipiente nas empresas brasileiras faz com que muitas vezes as empresas resolvam desafios tecnológicos, mas não registrem e/ou não sistematizem estas atividades, dificultando ou alongando o processo de levantamento de evidências para comprovação.

Por se tratar de uma metodologia inovadora, a Certics possui uma curva de aprendizagem. Nas avaliações já desenvolvidas observou-se que nas empresas onde a cultura de $\mathrm{P} \& \mathrm{D}$ e a inovação tecnológica estão amadurecidas, o entendimento dos resultados esperados da metodologia foi muito facilitado. Porém, naquelas que realizam atividades de $P \& D$ de uma forma quase tácita, ou que tenham forte assimetria nas áreas de competências, o entendimento dos objetivos e resultados esperados da Certics foi dificultado.

7 Pois a capacidade de desenvolver tecnologia e inovar é um extenso processo de aprendizagem (ver seção 3). 


\subsection{Estrutura operacional e evidências empíricas de aplicação da metodologia}

Para operacionalizar a aplicação da Certics em todo o território brasileiro, foi construída uma rede de dez parceiros acreditados para execução das avaliações Certics, conforme mostra a Figura 4. Para fins de disseminação dos conceitos e da lógica que embasam a metodologia Certics, foram realizados treinamentos de introdução à metodologia para o público interessado. Os treinamentos tinham por objetivo fornecer uma ideia geral sobre a metodologia. Foram realizados, ainda, treinamentos específicos para capacitação dos profissionais vinculados às entidades parceiras que atuariam como avaliadores líderes posteriormente. As sessões incluíram a exposição da metodologia Certics em detalhes, além de exercícios, provas e trabalhos conjuntos de análise de casos. ${ }^{8}$

\section{FIGURA 4}

Entidades avaliadoras Certics

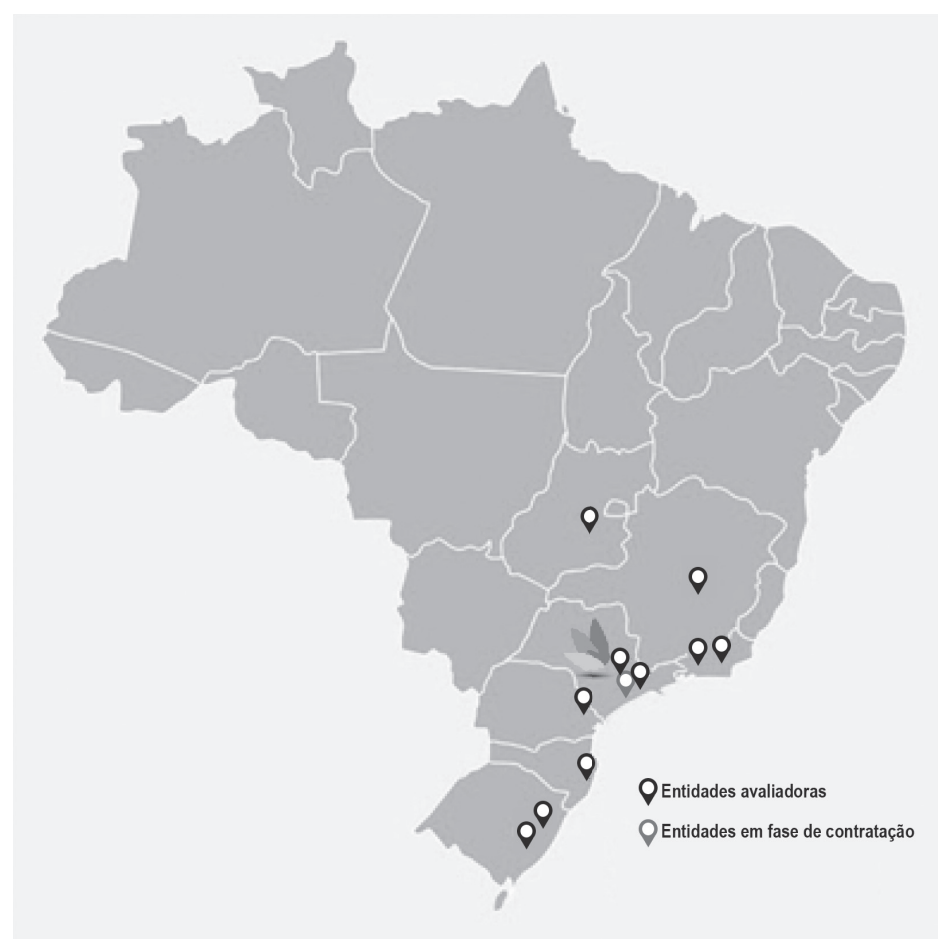

Fonte: Elaboração dos autores.

8 Até dezembro de 2014, já haviam sido realizadas 14 sessōes de treinamento, resultando na capacitação de 186 profissionais. Durante a execução dos treinamentos, diversas discussōes foram realizadas, o que contribuiu para o levantamento de pontos de interesse para o desenvolvimento e disseminação de orientaçôes adicionais sobre a metodologia Certics. 
Outra ação importante de suporte à operação foi o desenvolvimento de uma plataforma automatizada e flexível, dedicada ao processo de avaliação de modo geral, o CERTICSys. A plataforma é um aspecto fundamental em todo o processo, pois permite desde a simulação do uso da Certics, passando pelo registro de todas as informações e interação entre avaliadores e profissionais das empresas solicitantes da avaliação, até o acompanhamento dos resultados obtidos pós-certificação (monitoramento). O sistema de monitoramento possibilita o acompanhamento instantâneo de informaçōes relevantes para a gestão da operação, tais como número de softwares cadastrados na plataforma por fase do processo, características das organizaçôes solicitantes - porte, localização, segmento de atuação, etc. -, recursos financeiros previstos, desempenho das entidades que compõem a rede de avaliação, além do registro das melhorias na metodologia, entre outras.

Desde o início da operação Certics até fevereiro de 2015, foram avaliados e certificados 18 softwares e outros oito estão em fase de certificação, totalizando 22 empresas participantes (uma empresa certificou três softwares e uma empresa apresentou dois softwares para certificação).

No que tange aos resultados da metodologia Certics, verificou-se que até o momento a maior parte das empresas (43\%) encontra-se no Estado de São Paulo e Rio de Janeiro. Em relação ao tamanho das empresas, nota-se que há um destaque para aquelas de médio porte (46\%) e relativo equilíbrio na participação de micro (18\%), pequenas $(23 \%)$ e grandes empresas $(14 \%)$.

Com relação à motivação que levou estas empresas a obterem a certificação Certics, a que se destacou foi ampliação da competitividade do software em licitações públicas (56\%), seguida por associação da imagem do software no mercado como resultante de desenvolvimento e inovação tecnológica realizados no país (32\%).

No tocante a aspectos qualitativos preliminares, as empresas que passaram pelo processo de avaliação têm relatado ganhos obtidos no sentido de gerenciar melhor sua base de conhecimentos, em especial os relativos ao desenvolvimento tecnológico e inovação. O processo de pesquisa, investigação e resultados que levaram a inovações tecnológicas para atender um cliente, frequentemente, não eram registrados, nem considerados uma base a ser explorada para antecipar inovaçóes ou explorar novos nichos de mercado. Após o processo de avaliação, as empresas relataram ter maior entendimento dos resultados obtidos e identificar açōes para melhor sistematização destes resultados. 


\section{Considerações finais}

Neste trabalho foi apresentada a abordagem via formação de competências para identificar software com desenvolvimento tecnológico e inovação produzidos localmente e também a concretização desta abordagem na metodologia Certics. A aplicação dessa metodologia mostra alguns resultados preliminares importantes. No entanto, será necessário um período mais extenso para atestar que os objetivos mais amplos da política pública de incentivo ao desenvolvimento e inovação tecnológica para software estão sendo atendidos. E, mesmo sendo atendidos, entende-se que a política de preferência em compras públicas demanda outros fatores estruturantes e um ambiente que estimule o desenvolvimento setorial, para que uma cultura de $\mathrm{P} \& \mathrm{D}$ e inovação tecnológica seja efetivamente incorporada pelas empresas e se torne um fator competitivo estratégico.

Os objetivos governamentais de construção de um processo estruturado e rastreável de seleção de software, que atendesse a uma lógica rigorosamente embasada para obter resultados em prazos e custos viáveis para o mercado e governo, foram atendidos com a metodologia Certics. Verifica-se uma aceleração na curva de adoção da certificação Certics pelas empresas, havendo equilíbrio na participação de micro, pequenas e médias empresas, bem como na distribuição espacial das empresas participantes, que são indícios da viabilidade da Certics para atender a distintos portes e localizações geográficas.

Portanto, a abordagem via formação de competências e sua aplicação prática por meio da Metodologia Certics têm se mostrado útil para abordar software, mesmo em ambientes de baixa sistematização de atividades de P\&D e de inovação tecnológica, como o Brasil. Sua aplicação também tem permitido avaliar software de complexidades bastante distintas e em diversos setores da economia.

Ao se deslocar o foco de análise do software em si (linhas de código e funcionalidades) para as competências geradas em seu entorno, ganhou-se flexibilidade em diversos aspectos: diminui-se a necessidade do entendimento técnico específico do software avaliado. e o tempo de avaliação reduz-se drasticamente em relação a outras avaliações.

A abordagem via formação de competências pode ser adaptada para outros setores intensivos em tecnologia, pois as camadas mais altas da metodologia (ser resultante de desenvolvimento e inovação tecnológica locais e áreas de competências) têm um caráter genérico. A aplicação em outros setores demandaria uma adaptação dos resultados esperados e respectivas evidências. 
Porém, há uma dependência direta entre as áreas de competência definidas e a maturidade do setor em que é aplicada. Em setores de maior maturidade, podem ser necessárias outras áreas de competência para comprovar o desenvolvimento e inovações tecnológicas locais. A abordagem via formação de competência aqui concretizada na forma da metodologia Certics é específica para software que já foi desenvolvido e comercializado. Seu uso é praticamente inviável para projetos em andamento, como é o caso de muitas empresas startups. A Certics verifica, portanto, os ativos já gerados e aperfeiçoados pelo mercado, e não os que potencialmente podem ser criados. Todavia, percebe-se que a aplicação da avaliação no contexto das empresas pode contribuir para sinalizar melhorias potenciais em processos.

No atual estágio de maturidade do setor de software no Brasil, em que as competências empresariais ainda são pouco conhecidas e sistematizadas, esta abordagem poderá contribuir para mapear as capacidades efetivamente disponíveis no país.

Um impacto imediato verificado no uso desta abordagem é um amadurecimento na sistematização de atividades de P\&D\&I nas empresas de software, contribuindo para uma visão mais ampla e integrada dos requisitos e boas práticas para a realização de inovação, maior segurança no investimento público, decorrente da facilidade de monitoramento de resultados, e maior segurança na aquisição pública de software.

\section{Referências bibliográficas}

ALVES, A. M.; SALVIANO, C. F.; STEFANUTO, G.; MAINTINGUER, S. T.; MATTOS, C. V.; ZEITOUM, C.; MARTINEZ, M. R. M.; REUSS, G. CERTICS assessment methodology for software technological development and innovation. In: 9th INTERNATIONAL CONFERENCE ON THE QUALITY OF INFORMATION AND COMMUNICATIONS TECHNOLOGY. Quatic. Guimarães, Portugal, 2014, p. 174-177. DOI: 10.1109/ QUATIC.2014.32

BERG, P.; LEINONEM, M.; LEIVO, V.; PIHLAJAMAA, J. Assessment of quality and maturity level of R\&D. International Journal of Production Economics. v. 78, n. 1, p. 29-35, Jul. 2002.

BERG, P., PIHLAJAMAA, J.; NUMI, J. Measurement of the quality and maturity of the innovation process: methodology and case of a medium sized Finnish company. International Journal of Entrepreneurship and Innovation Management, v. 4, n. 4, p. 373-382, 2004.

CTI RENATO ARCHER. Metodologia de avaliação CERTICS para software: documento de definição. Relatório técnico TRT0012113. Campinas, v. 1.1, 2013. 
CHUNG, S. Korea improves quality of defense software. Korea IT Times, 28/03/2013. Disponível em: <http://www.koreaittimes.com/story/27466/korea-improves-quality-defense-software>. Acesso em: 02 ago. 2013.

CUNHA, N. C. As práticas gerenciais e suas contribuiçôes para a capacidade de inovação em empresas inovadoras. Tese (Doutorado). São Paulo: Universidade de São Paulo, Faculdade de Economia, Administração e Contabilidade, 2005

ENTERPRISE SPICE. Project Team, Enterprise SPICE (ISO/IEC 15504) an integrated model for Enterprise-wide improvement, technical report - Issue 1, September 2010. Disponível em: <www.enterprisespice.com>.

FLEURY, M. T.; FLEURY, A. Alinhando estratégia e competências. RAE-Revista de Administração de Empresas, v. 44, n. 1, p. 44-57. 2004.

. Construindo o conceito de competência. Revista de Administração Contemporânea, v. 5, n. especial, p. 183-196, 2001. Disponível em: <http://goo.gl/XtpwP>.

HWANG, S. M.; IM, S. Korean software process quality certification model. In: FIRST ACIS/JNU INTERNATIONAL CONFERENCE ON COMPUTERS, NETWORKS, SYSTEMS, AND INDUSTRIAL ENGINEERING. Jeju Island, Coreia, 2011.

IM, T. Information technology and organizational morphology: the case of the Korean Central Government. Public Administration Review, v. 71, n. 3, p. 435-443, 2011.

ISO/IEC - The International Organization for Standardization and the International Electrotechnical Commission. ISO/IEC 15504-2. Information technology - Process assessment - Part 2: Performing an assessment. 2003.

ISO/IEC 15504-7 - Information technology - Process assessment - Part 7: Assessment of organizational maturity. 2008.

JOUMARD, I. et al. Enhancing the effectiveness of public spending: experience in OECD Countries. França: OECD Publishing, 2004. (OECD Economics Department Working Papers, n. 380). Disponível em: <http://dx.doi.org/10.1787/355505224723>.

OMC - Organização Mundial do Comércio. Government Procurement: the plurilateral agreement. Overview of the Agreement on Government Procurement. Disponível em:

<http://www.wto.org/english/tratop_e/gproc_e/gpa_overview_e.htm>. Acesso em: 06 jan. 2014. PANDA, H.; RAMANATHAN, K. Technological capability assessment as an input for strategic planning: case studies at electricité du France and electricity generating authority of Thailand. Technovation, v. 17, n. 7), p. 359-390, 1997. 
Giancarlo Nuti Stefanuto, Angela Maria Alves, Clenio Figueiredo Salviano, Carolina Vaghetti Mattos, Camila Zeitoum

PRAHALAD, C. K.; HAMEL, G. The core competence of the corporation. Harvard Business Review, v. 68, n. 3, p. 79-91, 1990.

RUSH, H.; BESSANT, J.; HOBDAY, M. Assessing the technological capabilities of firms: developing a policy tool. R\&D Management, v. 37, n. 3, p. 221-236, 2007.

SALVIANO, C. F.; ALVES, A. M.; STEFANUTO, G.; MAINTINGUER, S. T.; MATTOS, C. V.; ZEITOUM, C. CERTICS - An ISO/IEC 15504 conformance model for software technological development and innovation. In: $14^{\text {th }}$ SPICE INTERNATIONAL CONFERENCE. Proc...Vilnius, Lithuania: CCIS 477, 4-6 November 2014, p. 48-59. DOI: 10.1007/2F978-3-319-13036-1_5.

SAXENIAN, A. Government and Guanxi: the chinese software industry in transition. London Business School, 2003 (DRC working papers, n. 19).

SEOK-KYOO, S. Introduction to TTA testing and certification laboratory. Korea IT Times, 27/09/2011. Disponível em: <http://www.koreaittimes.com/story/16988/introduction-tta-testing-and-certification-laboratory>. Acesso em: 02 ago. 2013.

SSENNOGA, F. Examining discriminatory procurement practices in developing countries. Journal of Public Procurement, v. 6, n. 3, p. 218-249, 2006.

SUNG-MI, K. TTA's software testing lab works towards the global market. Korea IT Times, 29/10/2012. Disponível em: <http://www.koreaittimes.com/story/24086/ttas-software-testing-lab-works-towards-global-market>. Acesso em 02 ago. 2013.

TEECE, D. Explicating dynamic capabilities: the nature and microfoundations of (sustainable) enterprise performance. Strategic Management Journal, n. 28, p. 1319-1350, 2007.

305, 1986.

. Profiting from Technological Innovation: Research Policy, v. 15, n. 6, p. 285-

USTR - United States Trade Representative. Turkey Trade Report, 2010. Disponível em: <www.ustr.gov/sites/default/files/uploads/reports/2010/NTE/2010_NTE_Turkey_final.pdf>. . National trade estimate report on foreign trade barriers. 2000. Disponível em: <www.ustr.gov/reports/nte/2000/contents.html>. 